\title{
High Resolution Radar Detection Based on Neutral Network
}

\author{
N. Pang \\ Beijing Institute of Technology, Beijing Institute of Radio Measurement, Beijing, China \\ P.J. Xu \\ Beijing Institute of Power Design, Beijing, China
}

\begin{abstract}
Neural Network has been broadly applied in the area of modern information technique including radar area. Traditional detection techniques can not detect objects accurately in High Resolution Radar system. Neural Network method can apperceive the tiny diversification of the object because of its flexible parallel processing structure and better mistake toleration. Back-Propagation method solved the problem of multiple network training. But this method is easy to shake and the convergence speed is slow. The Weight adjustment arithmetic based on momentum method can improve in the two aspects. The test shows the efficiency of this kind of adaptive BP method.
\end{abstract}

KEYWORD: High Resolution Radar; Neural Network; Adaptive BP method

\section{INTRODUCTION}

As a kind of intelligent technique, NN(Neural Network) as been broad applied in the area of modern information technique. The origin of neutral science can trace back to the 19th century, which is earlier than artificial intelligence. In recent years, people develop many artificial Neural Network model by simplification, abstraction and simulation. In 1975, Werbos proposed BP (Back-Propagation) training arithmetic which solved the problem of multiple network training[1].

HRR (High Resolution Radar) is a kind of new radar system which is studied and develops in 1980s. Its echo is from extended object which is different from "point" object echo[1]. Traditional detection techniques can not detect objects accurately in this case[5]. New methods need to be discovered. Neural Network has the characteristic of self-study, self-adaption and self-orgnization. It has unique capability to process data series. Especially, it can apperceive the tiny diversification of the object because of its flexible parallel processing structure and better mistake toleration. So it fits to dynamic and changeful environment of HRR.

\section{PRINCIPLE OF NEUTRAL NETWORK}

\subsection{Introduction of neutral network}

The basic idea of $\mathrm{NN}$ is really simple. Figure 1 shows a simple perceptron with multiple inputs and one output. A neuron is like a cell with various inputs and one scalar output. The neuron multiplys the inputs by synaptic weights and combines these products linearly to form the independent variable of the threshold function[2].

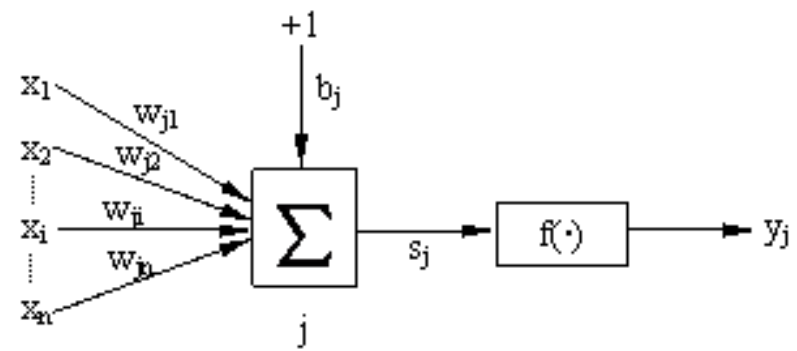

Figure 1. Simple NN principle

A NN is a highly interconnected set of small processors, each of which has a small memorization capability they learned from information submitted to them in the first place. Generally, the network is divided into layers and the parallel nature of NNs makes them well suited to work on multi parameter problems[2].

$s_{j}=\sum_{i=1}^{n} x_{i} w_{j i}+b_{j}=W_{j} X+b_{j}$,
$y_{j}=f\left(s_{j}\right)=f\left(\sum_{i=1}^{n} x_{i} w_{j i}+b_{j}\right)$

The output $y_{i}$ can be obtained by acting transfer function $f(\cdot)$ on the input $s_{i}$. 


\subsection{BP neutral network model}

The BP arithmetic is divided network learning into two phases. The first phase is inputting known learning sample. The output can be computed by network structure, weight and threshold set before from the first layer to the last layer. The second layer is modification of weight and thresh. The impact (grads) to the whole error can be computed from the last layer to the first layer. The weights and thresh can be modified by grads. The two phases process alternatively to convergence[3]. This method can be spread to network with multiple layers. BP network includes input layer, hidden-layer, and output layer. The model is composed of Input-output model, transfer function model, error computation model and self-learning model[7].

\section{INTRODUCTION OF DETECTION METHOD USING NN}

The reference [1] proposed the detection principle of using NN. The main idea of the author will be introduced in section 3.1 to 3.3. Section 3.4 gives the method in the case of multiple objects.

\subsection{Analyzation of HRR 1D image}

For LRR (Low Resolution Radar), the receiving signal $S_{R}(f)$ (DFT result) is identical with the transmitting signal $S_{T}(f)$.So the matched filter is the optimal filter for LRR. For HRR, the impulse response is $M(f)$. The receiving signal represents in type (2).

$$
S_{R}(f)=S_{T}(f) M(f)
$$

Then the matched filter characteristic of frequency spectrum is in type (3).

$$
H(f)=k S_{T}^{*}(t) M^{*}(f) e^{-j 2 \pi t_{0}}
$$

The matched filter is not only dependent on transmitting pulse but also on the characteristic of the target structure. It is not the best filter in this case. The detection of the $1 \mathrm{D}($ One-dimension) range image is a alternative analytical method[1]. Figure 2 is the 1D range image of one object[4].

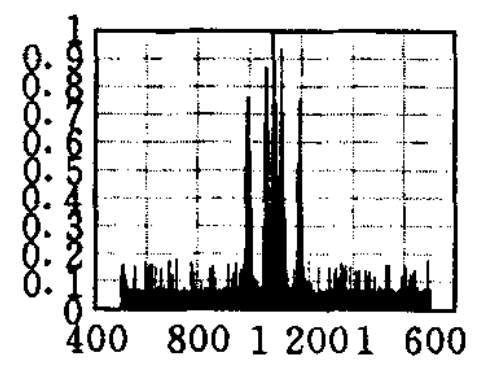

Figure 2. 1D range image of HRR echo

\subsection{The optimal perceptron neutral detector}

The aim of radar detection is to make sure the absence of a signal. Hence, the hypothesis testing problem can be stated as type (4).

$$
x(t)= \begin{cases}s(t)+c(t) & H_{1} \\ c(t) & H_{0}\end{cases}
$$

In type (1), $x(t)$ is the whole echo signal. $s(t)$ is the object echo signal. $n(t)$ is clutter. $H_{1}$ means the existence of object. $H_{0}$ means the absence of object. The detection problem comes down to the classification of two modes. The two kinds of modes can be implemented by training multiple layers sense sorter[1].

It is assumed that 1D range image of the object and clutter are $\left\{s_{i}, i=1,2, \cdots, N_{m}\right\}$ and $\left\{c_{i}, i=1,2, \cdots, N_{c}\right\}$. $N_{m}$ and $N_{c}$ are the number of the object signal sample and the clutter signal sample.

$$
s_{i}=\left[s_{1}^{(i)}, s_{2}^{(i)}, \cdots, s_{n}^{(i)}\right]^{T}, c_{i}=\left[c_{1}^{(i)}, c_{2}^{(i)}, \cdots, c_{n}^{(i)}\right]^{T}
$$

In the output end of the network, the anticipant output of the network is $d_{m}=1$ and $d_{c}=0$. The target function is defined as type (6).

$$
J(W)=\sum_{i=1}^{N_{m}}\left(d_{m}-y(i)\right)^{2}+\sum_{i=1}^{N_{c}}\left(d_{c}-y(i)\right)^{2}
$$

In type (6),

$$
y(i)=f\left(\sum_{j=1}^{h} w_{j}^{(2)} z_{j}^{(i)}\right), \quad W=\left\{w_{j i}^{(1)}, w_{j}^{(2)}\right\}
$$

It is assumed that the clutter distribution is Gauss model. The object and clutter is smooth random process. The detection probability and alarm probability are in type(8).

$$
\begin{gathered}
P_{D}=(2 \pi)^{-1 / 2} \int_{B_{1}}^{\infty} \exp \left(-z^{2} / 2\right) d z, \\
P_{F A}=(2 \pi)^{-1 / 2} \int_{B 2}^{\infty} \exp \left(-z^{2} / 2\right) d z
\end{gathered}
$$

Here, $B_{1}=\left(\eta_{0}-\mu_{0}\right) / \sigma_{0}, B_{2}=\eta_{0} / \sigma_{0}, \mu_{0}=\sum_{i=1}^{n} w_{i 1,1} m_{i}$ $\sigma_{0}=\sum_{i=1}^{n} w_{i, 1}^{2} \sigma_{i}^{2}, 0$

\subsection{The neutral detection of single object}

If $x_{i}$ represents the output of the $i$ th resolution unit. $x_{i}=1$ means over thresh. $x_{i}=0$ means below thresh. $\operatorname{Tr}=\{i \mid i \in M\}, M$ means point trace of one object. It is assumed the probability is $\alpha_{i}$, the united detection probability of $N$ independent resolution units represents in type (9) without objects[1]. 


$$
P_{n}\left(x_{1}, x_{2}, \cdots, x_{N}\right)=\prod_{i=0}^{N-1} \alpha_{i}^{x_{i}}\left(1-\alpha_{i}\right)^{1-x_{i}}
$$

In case of the existence of the object, it is assumed the detection probability of the $i$ th resolution unit is $\beta_{i}$, then judgement probability is below in type(10).

$$
P_{t}\left(x_{i} \mid x_{i} \in T r\right)=\prod_{i \in T r} \beta_{i}^{x_{i}}\left(1-\beta_{i}\right)^{1-x_{i}}
$$

The united condition probability using $\ln$ is below in type(11).

$$
\begin{aligned}
& L\left(x_{1}, x_{2}, \cdots, x_{N} / T r\right) \\
& =\ln \left[P_{t}\left(x_{1}, x_{2}, \cdots, x_{N} / T r\right) / P_{n}\left(x_{1}, x_{2}, \cdots, x_{N}\right)\right] \\
& =\sum_{i \in T r} x_{i} \ln \frac{\beta_{i}\left(1-\alpha_{i}\right)}{\alpha_{i}\left(1-\beta_{i}\right)}+\sum_{i \in T r} \ln \frac{\left(1-\beta_{i}\right)}{\left(1-\alpha_{i}\right)}
\end{aligned}
$$

The sufficient statistic is in type (12).

$$
R(x)=\sum_{i=0}^{N-1} w_{i} x_{i}
$$

The weight factor is in type(13).

$$
w_{i}= \begin{cases}\ln \frac{\beta_{i}\left(1-\alpha_{i}\right)}{\alpha_{i}\left(1-\beta_{i}\right)} & i \in T \\ 0 & i \notin T\end{cases}
$$

It is assumed the threshold is ${ }^{w_{0}}$, the detector can be implemented by mono-sensor. There are $\mathrm{N}$ neutral cells of the input layer and one neutral cell of the output layer. The function of the output node is step function.

$$
y=f\left(\sum_{i=0}^{N-1} w_{i} x_{i}-w_{0}\right)=\left\{\begin{array}{l}
1,\left(\sum_{i=0}^{N-1} w_{i} x_{i}-w_{0}\right)>0 \\
0,\left(\sum_{i=0}^{N-1} w_{i} x_{i}-w_{0}\right)<0
\end{array}\right.
$$

The 1D range image of the object and clutter are used respectively to train the neutral network, the anticipant output of the object is 1 , and the clutter is 0 . After training, the new sample data can be input to the neutral network. When the output is 1 , object exists. Whereas the output is 0 , the object is absent[1].

\subsection{The neutral detection of multiple objects}

For multiple objects, the neutral network model is applicable. In case of multiple objects, the runnable equation is below in type(15)[1].

$$
y_{j}=f\left(\sum_{i=0}^{N-1} w_{i j} x_{i}-w_{j}\right)=\left\{\begin{array}{l}
1,\left(\sum_{i=0}^{N-1} w_{i j} x_{i}-w_{j}\right)>0 \\
0,\left(\sum_{i=0}^{N-1} w_{i j} x_{i}-w_{j}\right)<0
\end{array}, j=1,2, \cdots, P\right.
$$

$P$ is the number of the objects. Figure 3 is $\mathrm{NN}$ of $P$ targets. In this case NN is a complex network with multiple inputs and multiple outputs .

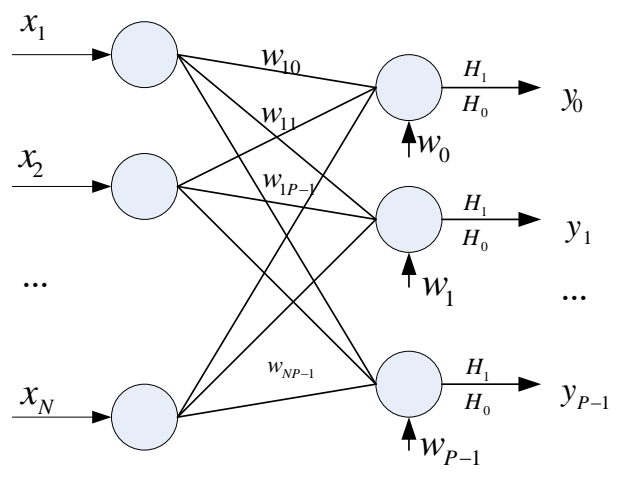

Figure 3. Neutral Network of multiple targets

\subsubsection{Estimation error analysis}

For $P$ group of samples, the whole error is in type(16).

$$
E=\frac{1}{2} \sum_{p=1}^{P} \sum_{j=1}^{m}\left(t_{j}^{p}-y_{j}^{p}\right)=\sum_{p=1}^{P} E_{p}
$$

$E_{p}$ is the error of the $p$ th sample.

$$
E_{p}=\frac{1}{2} \sum_{j=1}^{m}\left(t_{j}^{p}-y_{j}^{p}\right)
$$

$w_{j k}$ is adjusted according to the decrease trend of $E$.

$$
\Delta w_{j k}=-\eta \frac{\partial E}{\partial w_{j k}}=\sum_{p=1}^{P} \sum_{j=1}^{m} \eta\left(t_{j}^{p}-y_{j}^{p}\right) f^{\prime}\left(S_{j}\right) x_{k}
$$

\subsubsection{Adaptive BP method}

The normal BP arithmetic is a simple static searching excellence method. But this method is easy to shake and the convergence speed is slow. The Weight adjustment arithmetic based on momentum method can improve in the two aspects. The main idea is adding the portion of the weight adjustment last time to the weight adjustment of the current time [3].

$$
\begin{aligned}
& \Delta W(n)=\eta \Delta W(n-1)+\alpha(1-\eta) \frac{\partial E(n-1)}{\partial W(n-1)} \\
& W(n)=W(n-1)+\Delta W(n)
\end{aligned}
$$

$\alpha$ is momentum coefficient. In general, it is $0<\alpha<0.9 ; \eta$ is learning ratio. The range is between 0.001 and 10 . The momentum coefficient is damp item. This method decreases the shaking direction and increase the speed of learning.

\section{TEST AND ANALYZITION}

A group of echo series with multiple objects and 
without objects are selected to train BP network. The trained sample number of the two models is half of the whole number. The main parameters are shown in table 1.

Table 1. Comparison between with and without optimization

\begin{tabular}{|l|l|l|}
\hline Main parameter & Classical BP & Adaptive BP \\
\hline Training sample Num. & $100(50$ of each model $)$ & 100 \\
\hline Testing sample Num. & 100 & 100 \\
\hline Training times & 1000 & 1000 \\
\hline Network structure & Single Layer & Single Layer \\
\hline Error goal & 0.001 & 0.001 \\
\hline Learning ratio & 0.003 & 0.003 \\
\hline Momentum constant & - & 0.6 \\
\hline
\end{tabular}

Figure 4 is the result of $\mathrm{BP}$ training. It is easy to know the classical BP method shakes up and down. The convergence speed is slower. But the adaptive method has smooth performance curve and faster convergence speed.

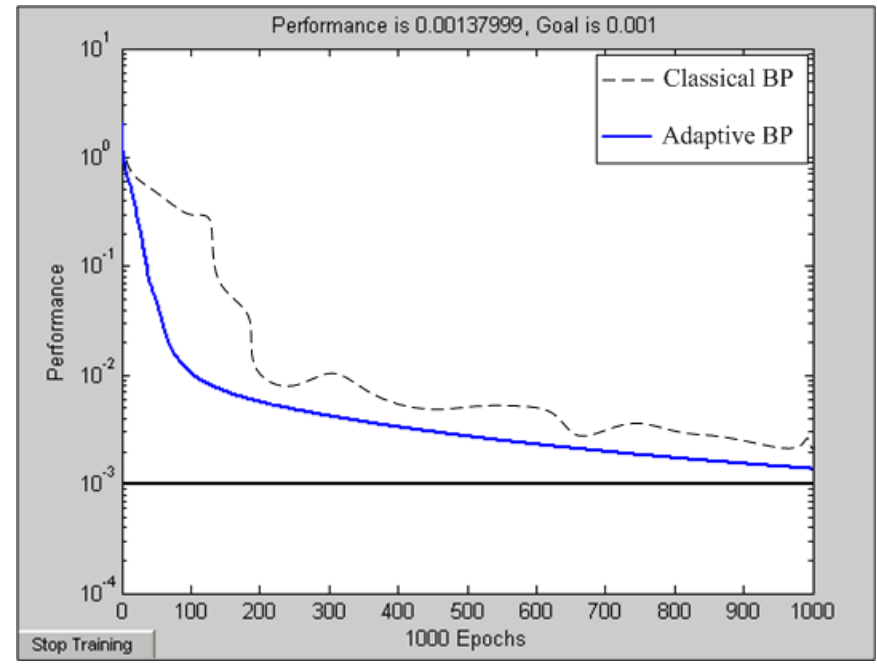

Figure 4. Performance comparesion of two BP methods

\section{CONCLUSION}

An attempt has been made to detect the object by NN. The normal BP arithmetic is easy to shake and the convergence speed is slow. The Adaptive BP method can solve the two problems. This advantage is important in many applications, especially when real-time online adjustment of the parameters or weights is needed.

\section{REFERENCES}

[1] Huang De Shuang.2001.Intelligent Signal Processing Technique for High Resolution Radars.Beijing:China Machine Press.

[2] UlanovaE.A.,NesterukD.A.2008. Neutral network detection. 2008 International Conference Modern Technique and Technologies:122-125.

[3] Guo Zunhua,Li Shaohong.2008. Performance Simulation of Recognition of Radar Target Range Profile based on BP Neural Network. Modem Radar 30(1):17-25.

[4] Liu Bing,Luo Dingli.2011.Study on Target Detection Method of High Resolution Radar.Fire Control Radar Technology 40(4):56-60.

[5] Yu Hongbo,Wang Guohong,Wang Na.2011.A Simulation Method of Extended Targets Echo Based on Highresolution Radar.Fire control and Command Control 36(6):138-140.

[6] James T. Lo and Justin Nave.2007.Adaptive Neural Filters with Fixed Weights. Proceedings of International Joint Conference on Neural Network. Orlando, Florida, USA, August 12-17.

[7] Lin Yun, Li Jing-chao. 2011. Radar Signal Recognition Algorithms Based on Neural Network and Grey Relation Theory.Cross Strait Quad-Regional Radio Science and Wireless Technology Conference. 